\title{
The Multifrequency Spectral Eulerian (MUSE) Model of a Traveling Wave Tube
}

\author{
John G. Wöhlbier, Student Member, IEEE, John H. Booske, Senior Member, IEEE, and \\ Ian Dobson, Senior Member, IEEE
}

\begin{abstract}
We derive from Eulerian electron beam equations the multifrequency spectral Eulerian (MUSE) model, a new onedimensional (1-D) nonlinear multifrequency model of a traveling wave tube (TWT). We also derive from the same equations a Lagrangian "disk" model, LATTE, so that MUSE may be directly compared to a Lagrangian approach. The models are compared to the large signal code Christine 1-D on a set of TWT parameters which are based on a single section of the Hughes $8537 \mathrm{H}$ L-band TWT. Aspects of the physics, nonlinearities, and simulation dimensions of the MUSE model are discussed, as well as its relation to the method of collective variables. A simplified MUSE model S-MUSE useful for analysis is also presented and its applications are discussed.
\end{abstract}

Index Terms-Collective variables, Eulerian, Lagrangian, multifrequency, nonlinear, traveling wave tube (TWT).

\section{INTRODUCTION}

$\mathbf{T}$ RAVELING wave tubes (TWTs) continue to find widespread application due to their inherently wide bandwidths and their high-frequency high-power operating points. A compromising feature of the TWT (and the solid state amplifier) is the nonlinear behavior of the device. The nonlinearity manifests as a saturating mechanism and spectral distortion. Both of these effects limit the efficiency of the TWT. For electronic countermeasures, distortions put power into harmonics with a concomitant, undesired reduction of power at the intended drive frequency. For digital communications applications, the distortions increase bit error rates, and this limits data rates.

Reducing nonlinear distortions in TWTs would increase efficiency of electronic countermeasure systems, and would increase data rates and efficiency in digital communications applications. These objectives provide strong motivation for approaches to understand nonlinear distortion mechanisms in TWTs.

The nature of the distortion in TWTs is "intermodulation distortion." For steady-state input signals with multifrequency content, an intermodulation product of order $K$ for frequencies $f_{1}, f_{2}, \ldots, f_{P}$ is of the form

$$
r_{1} f_{1}+r_{2} f_{2}+\cdots+r_{P} f_{P}
$$

Manuscript received November 6, 2001; revised February 8, 2002. This work was supported in part by the U.S. Air Force Office of Scientific Research (AFOSR) under Grant 49620-00-1-0088 and in part by DUSD (S\&T) under the Innovative Microwave Vacuum Electronics Multidisciplinary University Research Initiative (MURI) program, managed by the AFOSR under Grant F49620-99-1-0297.

The authors are with the ECE Department, the University of Wis consin-Madison, Madison, WI 53706 USA (e-mail: booske@engr.wisc.edu).

Digital Object Identifier 10.1109/TPS.2002.801603 where $r_{j}$ are integers (possibly zero) and

$$
K=\left|r_{1}\right|+\left|r_{2}\right|+\cdots+\left|r_{P}\right| \text {. }
$$

This structure also includes harmonic frequencies if all but one of the $r_{j}$ are zero. For a given set of steady-state input frequencies, a nonlinear multifrequency TWT model will predict evolution of circuit power at the input, or drive, frequencies as well as the intermodulation frequencies produced by the drive frequencies.

Nonlinear modeling of steady-state inputs to TWTs has been extensively studied with models that use Lagrangian coordinates rather than Eulerian coordinates for the electron beam equations. Six years after Pierce's seminal Eulerian linear theory [1], Nordsieck developed the Lagrangian nonlinear theory to account for charge overtaking in the electron beam and saturation of the circuit field [2]. The first multifrequency TWT models [3], [4] also used the Lagrangian beam description so that they too would predict saturation. A modern multifrequency code using such a Lagrangian description is Christine 1-D [5], [6].

In this paper, we derive a new one-dimensional (1-D) nonlinear multifrequency Eulerian TWT model, the multifrequency spectral Eulerian (MUSE) model. We also derive a Lagrangian "disk" model, Lagrangian TWT Equations (LATTE), from the same initial equations for comparison purposes as well as to demonstrate the theoretical relation between MUSE and a disk model. A simplified MUSE model, S-MUSE, more suitable for analysis is also derived. These three models are compared to each other and Christine 1-D for a set of TWT parameters which are based on the Hughes (now Boeing) 8537H L-band TWT design. The comparison to Christine 1-D is particularly useful since this code is widely known and used, and it has been validated against experiment for the Hughes TWT [7], [8]. The nonlinearities of MUSE and LATTE are compared, and an example of how MUSE can examine fundamental distortion mechanisms is provided. We also study how the dimensions of the MUSE model and LATTE scale with number of frequencies, an important issue for assessing the use of MUSE as a numerical tool. Next, we discuss the relation of the MUSE model to the "method of collective variables" in free-electron laser theory [9]. Lastly, we briefly indicate results that come from analysis of the S-MUSE model.

Some of the early work considering nonlinear effects involving more than one frequency in TWTs was done using Eulerian coordinates. Putz [10] considered two frequencies and studied cross-modulation. Curtice [11] extended this work to include space charge and finite Pierce gain parameter $C$. Sobol 
[12] and DeGrasse [13] considered two input frequencies and their harmonics. The group of Datta et al. have published a collection of articles using an approximate third order nonlinear multifrequency Eulerian TWT model [14]-[16]. Their work focuses on approximate analytic solutions to cases with a small number of input frequencies, including studies of harmonic generation and harmonic injection. The complexity of their approximate solutions increases substantially with increasing numbers of tones. In contrast, the MUSE model is a system of first order ordinary differential equations that can in principle handle an arbitrary number of tones. MUSE is the first "exact" steady-state nonlinear Eulerian model in that it makes no approximations in the nonlinearities. The S-MUSE model is an approximate model that makes different approximations than other models in the literature.

The fundamental Eulerian equations used to derive MUSE are similar to those Pierce used for his linear single frequency theory. However, our equations are nonlinear and accommodate signals with multifrequency content. Our starting equations are the same as the equations used by Datta et al. except that where we use the continuity equation, they use an integral of the continuity equation by assuming that $\nabla \times \mathbf{B}=0$ for perturbed quantities in Ampere's law.

Section II presents the models to be considered. We derive the MUSE model and discuss its numerical solution, discuss the disk model LATTE whose derivation is given in Appendix I, and derive the S-MUSE model. The models are compared to each other and Christine 1-D in Section III. In particular, we look at power versus axial distance, a constant of the motion and the issue of electron overtaking. Section IV discusses the nonlinearities in MUSE, the dimensional dependence of MUSE and LATTE on simulation parameters, and the relation of MUSE to the "collective variable" theory of free electron lasers. Section V indicates the applications of S-MUSE and Section VI concludes the paper.

\section{TWT MODELS}

\section{A. MUSE}

1) Derivation: For the MUSE model, the helix is modeled as a lossless transmission line ${ }^{1}$ and Eulerian equations are used for the electron beam. In particular, the time domain model equations are

$$
\begin{aligned}
\frac{\partial V}{\partial z} & =h_{1} * \frac{\partial I}{\partial t} \\
\frac{\partial I}{\partial z} & =h_{2} * \frac{\partial V}{\partial t}-A \frac{\partial \rho}{\partial t} \\
\frac{\partial E}{\partial z} & =\frac{\rho}{\epsilon_{0}} \\
\frac{\partial v}{\partial t}+v \frac{\partial v}{\partial z} & =-\frac{e}{m_{e}} h_{1} * \frac{\partial I}{\partial t}+\frac{e}{m_{e}} R * E \\
\frac{\partial \rho}{\partial t}+v \frac{\partial \rho}{\partial z} & =-\rho \frac{\partial v}{\partial z}
\end{aligned}
$$

${ }^{1}$ Losses are incorporated in all of the models in the code LATTE/MUSE Numerical Suite. The code is available at http://lmsuite.org. where $z$ is axial distance, $t$ is time, $V$ is transmission line voltage, $I$ is transmission line current, $E$ is the space charge electric field, $v$ is electron beam velocity, and $\rho$ is the volume charge density of an electron beam with cross-sectional area $A$. The $*$ denotes convolution and this allows for frequency dependence of circuit and beam parameters. The functions $h_{1}$, $h_{2}$, and $R$ are the inverse Fourier transforms

$$
\begin{aligned}
& h_{1}(z, t)=\mathcal{F}^{-1}\left\{\frac{\tilde{K}\left(z, f_{\ell} \omega_{0}\right)}{\tilde{v}_{\mathrm{ph}}\left(z, f_{\ell} \omega_{0}\right)}\right\} \\
& h_{2}(z, t)=\mathcal{F}^{-1}\left\{\frac{1}{\tilde{K}\left(z, f_{\ell} \omega_{0}\right) \tilde{v}_{\mathrm{ph}}\left(z, f_{\ell} \omega_{0}\right)}\right\} \\
& R(z, t)=\mathcal{F}^{-1}\left\{\tilde{R}\left(z, f_{\ell} \omega_{0}\right)\right\}
\end{aligned}
$$

where the functions $\tilde{K}\left(z, f_{\ell} \omega_{0}\right), \tilde{v}_{\mathrm{ph}}\left(z, f_{\ell} \omega_{0}\right)$, and $\tilde{R}\left(z, f_{\ell} \omega_{0}\right)$ are frequency domain circuit interaction impedance [17], cold circuit phase velocity, and space charge reduction factor [18], respectively. The inverse transforms are aperiodic functions of $t$ and are functions of $z$ to allow for spatial variation of circuit parameters. In the remainder of the paper, notation of this $z$ dependence is suppressed. The constants $e, m_{e}$, and $\epsilon_{0}$ are electron charge, electron mass, and permittivity of free space, respectively.

For reasons that will be made clear later, we first make the coordinate transformation

$$
\left[\begin{array}{l}
z \\
\psi
\end{array}\right]=\left[\begin{array}{cc}
1 & 0 \\
\frac{\omega_{0}}{u_{0}} & -\omega_{0}
\end{array}\right]\left[\begin{array}{l}
z \\
t
\end{array}\right] .
$$

Then

$$
\begin{aligned}
\frac{\partial V}{\partial z} & =-\frac{\omega_{0}}{u_{0}} \frac{\partial V}{\partial \psi}-\omega_{0} h_{1} * \frac{\partial I}{\partial \psi} \\
\frac{\partial I}{\partial z} & =-\omega_{0} h_{2} * \frac{\partial V}{\partial \psi}-\frac{\omega_{0}}{u_{0}} \frac{\partial I}{\partial \psi}+A \omega_{0} \frac{\partial \rho}{\partial \psi} \\
\frac{\partial E}{\partial z} & =-\frac{\omega_{0}}{u_{0}} \frac{\partial E}{\partial \psi}+\frac{\rho}{\epsilon_{0}} \\
v \frac{\partial v}{\partial z} & =\frac{\omega_{0} e}{m_{e}} h_{1} * \frac{\partial I}{\partial \psi}+\frac{e}{m_{e}} R * E+\omega_{0}\left(1-\frac{v}{u_{0}}\right) \frac{\partial v}{\partial \psi} \\
v \frac{\partial \rho}{\partial z} & =\omega_{0}\left(1-\frac{v}{u_{0}}\right) \frac{\partial \rho}{\partial \psi}-\rho\left(\frac{\partial v}{\partial z}+\frac{\omega_{0}}{u_{0}} \frac{\partial v}{\partial \psi}\right)
\end{aligned}
$$

We assume all inputs to the system (signals at $z=0$ ) are periodic in $t$ with fundamental frequency $\omega_{0}$. This implies that solutions as functions of $(z, t)$ are periodic in $t$ with fundamental period $2 \pi / \omega_{0}$ and that solutions as functions of $(z, \psi)$ are periodic in $\psi$ with fundamental period $2 \pi$.

For a function $x(z, \psi)$ periodic in $\psi$ we use the Fourier series relations

$$
\begin{aligned}
x(z, \psi) & =\sum_{\ell=-\infty}^{\infty} \tilde{x}_{\ell}(z) e^{i f_{\ell} \psi} \\
\tilde{x}_{\ell}(z) & =\frac{1}{2 \pi} \int_{2 \pi} x(z, \psi) e^{-i f_{\ell} \psi} d \psi
\end{aligned}
$$

where the $f_{\ell}$ are integers indexed by $\ell$. The set of frequencies $\left\{f_{\ell}\right\}$ is chosen to be the frequencies with nonzero Fourier coefficients, thus $\left\{f_{\ell}\right\}$ is the drive frequencies together with the 
frequencies produced from nonlinear interactions. We index the frequencies so that $f_{-\ell}=-f_{\ell}$ and $f_{m}>f_{n}$ for $m>n$. Since our functions are real valued

$$
\tilde{x}_{-\ell}=\tilde{x}_{\ell}^{*}
$$

Computing Fourier coefficients of (10)-(14) gives the MUSE model

$$
\begin{aligned}
& \frac{d \tilde{V}_{\ell}}{d z}=-\frac{i f_{\ell} \omega_{0}}{u_{0}} \tilde{V}_{\ell}-\frac{i f_{\ell} \omega_{0} \tilde{K}\left(f_{\ell} \omega_{0}\right)}{\tilde{v}_{\mathrm{ph}}\left(f_{\ell} \omega_{0}\right)} \tilde{I}_{\ell} \\
& \frac{d \tilde{I}_{\ell}}{d z}=-\frac{i f_{\ell} \omega_{0}}{\tilde{K}\left(f_{\ell} \omega_{0}\right) \tilde{v}_{\mathrm{ph}}\left(f_{\ell} \omega_{0}\right)} \tilde{V}_{\ell}-\frac{i f_{\ell} \omega_{0}}{u_{0}} \tilde{I}_{\ell}+i f_{\ell} \omega_{0} A \tilde{\rho}_{\ell} \\
& \frac{d \tilde{E}_{\ell}}{d z}=-\frac{i f_{\ell} \omega_{0}}{u_{0}} \tilde{E}_{\ell}+\frac{\tilde{\rho}_{\ell}}{\epsilon_{0}} \\
& \sum_{\substack{m, n \\
f_{m}+f_{n}=f_{\ell}}} \tilde{v}_{m} \frac{d \tilde{v}_{n}}{d z} \\
& =\frac{i f_{\ell} \omega_{0} e \tilde{K}\left(f_{\ell} \omega_{0}\right)}{m_{e} \tilde{v}_{\mathrm{ph}}\left(f_{\ell} \omega_{0}\right)} \tilde{I}_{\ell}+\frac{e}{m_{e}} \tilde{R}\left(f_{\ell} \omega_{0}\right) \tilde{E}_{\ell}+i f_{\ell} \omega_{0} \tilde{v}_{\ell} \\
& -\sum_{\substack{m, n \\
f_{m}+f_{n}=f_{\ell}}} \frac{i f_{n} \omega_{0}}{u_{0}} \tilde{v}_{m} \tilde{v}_{n} \\
& \sum_{\substack{m, n \\
f_{m}+f_{n}=f_{\ell}}} \tilde{v}_{m} \frac{d \tilde{\rho}_{n}}{d z} \\
& =i f_{\ell} \omega_{0} \tilde{\rho}_{\ell}-\frac{i f_{\ell} \omega_{0}}{u_{0}} \sum_{\substack{m, n \\
f_{m}+f_{n}=f_{\ell}}} \tilde{v}_{m} \tilde{\rho}_{n}-\sum_{\substack{m, n \\
f_{m}+f_{n}=f_{\ell}}} \frac{d \tilde{v}_{m}}{d z} \tilde{\rho}_{n}
\end{aligned}
$$

where $-\infty \leq \ell \leq \infty$. We have used that for $x(z, \psi)$ and $y(z, \psi)$ periodic, multiplication becomes convolution

$$
x(z, \psi) y(z, \psi) \stackrel{\mathcal{F}}{\longrightarrow} \sum_{\substack{m, n \\ f_{\ell}=f_{m}+f_{n}}} \tilde{x}_{m}(z) \tilde{y}_{n}(z) .
$$

The summation notation should be read as "sum over integers $m$ and $n$ such that $f_{m}+f_{n}=f_{\ell}$."

2) Method of Numerical Solution: For practical implementation one neglects higher frequencies and limits $\ell$ to $-M \leq$ $\ell \leq M$. Then, the MUSE model has $5(2 M+1)$ complex equations.

During integration of the MUSE model, one needs to solve (21) and (22) for the derivatives $d \tilde{v}_{\ell} / d z$ and $d \tilde{\rho}_{\ell} / d z$. Equations (21) and (22) for $-M \leq \ell \leq M$ are the linear systems

$$
\begin{aligned}
& \mathbf{S w}_{v}=\mathbf{b}_{v} \\
& \mathbf{S w}_{\rho}=\mathbf{b}_{\rho}
\end{aligned}
$$

where $\mathbf{w}_{v}, \mathbf{w}_{\rho}, \mathbf{b}_{v}$, and $\mathbf{b}_{\rho}$ are $2 M+1$ vectors and $\mathbf{S}$ is a $(2 M+$ $1) \times(2 M+1)$ matrix. The $\ell$ th entries of $\mathbf{w}_{v}$ and $\mathbf{w}_{\rho}$ are $d \tilde{v}_{\ell} / d z$ and $d \tilde{\rho}_{\ell} / d z$, respectively, the $\ell$ th entries of $\mathbf{b}_{v}$ and $\mathbf{b}_{\rho}$ are equal to the right-hand sides of (21) and (22), respectively, and the $\ell$ th row and $n$th column entry of $\mathbf{S}$ is $\tilde{v}_{m}$ where $f_{m}+f_{n}=f_{\ell}$.

We choose the relation between the initial value of the circuit current and the initial value of the circuit voltage as

$$
\tilde{I}_{\ell}(0)=-\frac{\tilde{V}_{\ell}(0)}{\tilde{K}\left(f_{\ell} \omega_{0}\right)} .
$$

One can show for the linearization of (18)-(22) that, up to a very small correction, (23) ensures that only the three forward waves of Pierce theory are excited [19]. If one includes modulations on beam quantities, (23) is still the appropriate relation between initial voltage and initial current so that only forward waves are excited.

The circuit power at angular frequency $\omega$ is due to both the positive and negative frequencies, so

$$
P_{\omega}(z)=-\left[\tilde{V}_{\ell}(z) \tilde{I}_{\ell}^{*}(z)+\tilde{V}_{\ell}^{*}(z) \tilde{I}_{\ell}(z)\right] .
$$

The negative sign in (24) is due to the form of the telegrapher equations which are chosen to be consistent with [19]. Given input power $P_{\omega}(0)$ and phase $\phi_{\omega}(0)$ at $\omega=f_{\ell} \omega_{0}>0$, the initial value of circuit voltage is

$$
\begin{aligned}
\tilde{V}_{\ell}(0) & =\sqrt{\frac{P_{\omega}(0) \tilde{K}\left(f_{\ell} \omega_{0}\right)}{2}} e^{i \phi_{\omega}(0)} \\
\tilde{V}_{-\ell}(0) & =\tilde{V}_{\ell}^{*}(0) .
\end{aligned}
$$

If there are (periodic) modulations on any of the quantities at the input, one can calculate the proper initial values using (16). Otherwise, for $\ell \neq 0, \tilde{E}_{\ell}=\tilde{v}_{\ell}=\tilde{\rho}_{\ell}=0$. Also, one has $\tilde{v}_{0}(0)=u_{0}, \tilde{\rho}_{0}(0)=\rho_{0}$, and $\tilde{V}_{0}=\tilde{I}_{0}=\tilde{E}_{0}=0$.

By treating this problem as an initial value problem with the described initial conditions, we are assuming a perfectly matched load and no reflections. When a sever and a mismatched load are included, one must treat the problem as a boundary value problem and use an iterative scheme such as a shooting method [20, Ch. 17]. In the boundary value problem, the relation of circuit voltage to circuit current at the input [cf. (23)] will be determined as part of the solution to be consistent with the reflections.

\section{B. LATTE}

Appendix I derives LATTE starting from (10)-(14), which are the same equations used to derive the MUSE model. Comparable multifrequency disk models are well known, e.g. [3]-[5], [21], but the derivation in Appendix I is unique in the way it represents the method of characteristics as a general transformation between Eulerian and Lagrangian coordinates.

The transformation reveals interesting information about the relation between MUSE and LATTE. For example, the Lagrangian continuity equation [(46)] is often written as (see, e.g., $[17$, p. 302])

$$
I d t=I_{0} d t_{0}
$$

where $I$ is beam current, $t$ is time, $I_{0}$ is initial beam current, and $t_{0}$ is the Lagrangian initial time. However, it has not been pointed out in the microwave device literature that

$$
\frac{d t}{d t_{0}}=\frac{I_{0}}{I}
$$

is the Jacobian of the transformation from Lagrangian to Eulerian coordinates. We use this fact in Section III-C to examine when electron overtaking occurs. Appendix I also identifies a constant of the motion for MUSE and LATTE given in (53). 


\section{S-MUSE}

1) Derivation: A simplification of MUSE results in the S-MUSE model. S-MUSE is easier to analyze than MUSE while it retains important nonlinear physics. The simplifications are

1) approximating $\tilde{\rho}_{0}(z)$ and $\tilde{v}_{0}(z)$ as constants $\rho_{0}$ and $u_{0}$;

2) neglecting the ac portion of velocity in the convective derivative, i.e., letting $v(\partial / \partial z) \approx u_{0}(\partial / \partial z)$;

3 ) ignoring nonlinearities higher than second order in the continuity equation.

Since we have made the transformation $(z, t) \rightarrow(z, \psi)$, some of the nonlinearity of the convective derivative is retained, i.e., the original $v(\partial / \partial z)$ term [as seen in (4) and (5)] splits into $v(\partial / \partial z)+v\left(\omega_{0} / u_{0}\right)(\partial / \partial \psi)$ and we only linearize the first of these two nonlinear terms in (13) and (14). This is the motivation for introducing the $(z, \psi)$ coordinates in (9). The S-MUSE model is

$$
\begin{aligned}
\frac{d \tilde{V}_{\ell}}{d z} & =-\frac{i f_{\ell} \omega_{0}}{u_{0}} \tilde{V}_{\ell}-\frac{i f_{\ell} \omega_{0} \tilde{K}\left(f_{\ell} \omega_{0}\right)}{\tilde{v}_{\mathrm{ph}}\left(f_{\ell} \omega_{0}\right)} \tilde{I}_{\ell} \\
\frac{d \tilde{I}_{\ell}}{d z} & =-\frac{i f_{\ell} \omega_{0}}{\tilde{K}\left(f_{\ell} \omega_{0}\right) \tilde{v}_{\mathrm{ph}}\left(f_{\ell} \omega_{0}\right)} \tilde{V}_{\ell}-\frac{i f_{\ell} \omega_{0}}{u_{0}} \tilde{I}_{\ell}+i f_{\ell} \omega_{0} A \tilde{\rho}_{\ell}
\end{aligned}
$$

$$
\frac{d \tilde{E}_{\ell}}{d z}=-\frac{i f_{\ell} \omega_{0}}{u_{0}} \tilde{E}_{\ell}+\frac{\tilde{\rho}_{\ell}}{\epsilon_{0}}
$$

$$
\begin{aligned}
\frac{d \tilde{v}_{\ell}}{d z}= & \frac{i f_{\ell} \omega_{0} e \tilde{K}\left(f_{\ell} \omega_{0}\right)}{m_{e} u_{0} \tilde{v}_{\mathrm{ph}}\left(f_{\ell} \omega_{0}\right)} \tilde{I}_{\ell}+\frac{e}{m_{e} u_{0}} \tilde{R}\left(f_{\ell} \omega_{0}\right) \tilde{E}_{\ell} \\
& -\frac{1}{u_{0}^{2}} \sum_{\substack{m \neq 0, n \neq 0 \\
f_{m}+f_{n}=f_{\ell}}} i f_{n} \omega_{0} \tilde{v}_{m} \tilde{v}_{n}
\end{aligned}
$$

$$
\begin{aligned}
\frac{d \tilde{\rho}_{\ell}}{d z}= & -\frac{i f_{\ell} \omega_{0} e \rho_{0} \tilde{K}\left(f_{\ell} \omega_{0}\right)}{m_{e} u_{0}^{2} \tilde{v}_{\mathrm{ph}}\left(f_{\ell} \omega_{0}\right)} \tilde{I}_{\ell}-\frac{e \rho_{0}}{m_{e} u_{0}^{2}} \tilde{R}\left(f_{\ell} \omega_{0}\right) \tilde{E}_{\ell} \\
& -\frac{i f_{\ell} \omega_{0} \rho_{0}}{u_{0}^{2}} \tilde{v}_{\ell}-\frac{e}{m_{e} u_{0}^{2}} \sum_{\substack{m \neq 0, n \neq 0 \\
f_{m}+f_{n}=f_{\ell}}} \frac{i f_{m} \omega_{0} \tilde{K}\left(f_{m} \omega_{0}\right)}{\tilde{v}_{\mathrm{ph}} f_{m} \omega_{0}} \\
& \cdot \tilde{I}_{m} \tilde{\rho}_{n}-\frac{e}{m_{e} u_{0}^{2}} \sum_{\substack{m \neq 0, n \neq 0 \\
f_{m}+f_{n}=f_{\ell}}} \tilde{R}\left(f_{m} \omega_{0}\right) \tilde{E}_{m} \tilde{\rho}_{n} \\
& +\frac{\rho_{0}}{u_{0}^{3}} \sum_{\substack{m \neq 0, n \neq 0 \\
f_{m}+f_{n}=f_{\ell}}} i f_{n} \omega_{0} \tilde{v}_{m} \tilde{v}_{n}-\frac{i f_{\ell} \omega_{0}}{u_{0}^{2}} \sum_{\substack{m \neq 0, n \neq 0 \\
f_{m}+f_{n}=f_{\ell}}} \tilde{v}_{m} \tilde{\rho}_{n}
\end{aligned}
$$

where $-M \leq \ell \leq M, \ell \neq 0$.

2) Vector Form: The S-MUSE model (25)-(29) may be written in the following vector form that is particularly useful for analysis:

$$
\dot{\mathrm{x}}=\mathbf{A x}+\mathbf{H}(\mathrm{x}, \mathrm{x})
$$

where $\mathbf{x}$ is a 5(2M)-dimensional complex vector, $\mathbf{A}$ is a $5(2 M) \times 5(2 M)$ complex matrix, $\mathbf{H}$ is a $5(2 M) \times 5(2 M) \times 5(2 M)$ complex 3-tensor, and overdot represents $d / d z$. The detailed specification and indexing of $\mathbf{x}$, $\mathbf{A}$, and $\mathbf{H}$ as well as a formula to compute the components of $\dot{\mathrm{x}}$ are found in Appendix II.
TABLE I

8537H PARAMETERs (CONSTANT PITCH SeCtion)

\begin{tabular}{lc} 
Parameter & Model Value \\
\hline Helix mean radius & $0.2353 \mathrm{~cm}$ \\
Helix wire width & $0.0305 \mathrm{~cm}$ \\
Pitch & $0.13 \mathrm{~cm}$ \\
Cathode voltage & $3.1 \mathrm{kV}$ \\
Beam current & $65.5 \mathrm{~mA}$ \\
Min. beam radius & $0.0962 \mathrm{~cm}$ \\
BN $\left(\leq_{r}=5.4\right)$ support rod & 1.21 \\
smeared permittivity & \\
\hline
\end{tabular}

Equation (30) shows the S-MUSE model in vector form as a sum of a linear term and a quadratic nonlinearity. In this form, they are well suited for a type of perturbation theory where the zeroth order solution is that of a linear system.

\section{NUMERICAL EXAMPLE}

In this section, we consider a numerical example comparing the models amongst themselves and Christine 1-D. First, we look at circuit power as a function of axial distance. We use LATTE as a benchmark and present deviations in decibels of the other models from LATTE. For the simulations, we also check the constant of the motion (53) and compare the terms making up the constant of the motion. For the case of one drive frequency, we consider the question of electron overtaking and its correlation to the deviation of MUSE from LATTE. Lastly, we discuss practical issues concerning the choice of frequencies for a simulation comparison with Christine 1-D.

For our numerical study, we simulate one constant pitch section of the 8537H TWT with no sever or circuit loss. The parameters for the $8537 \mathrm{H}$ are taken from [7] and are shown in Table I. For the frequency dependent parameters $\tilde{v}_{\mathrm{ph}}\left(f_{\ell} \omega_{0}\right), \tilde{K}\left(f_{\ell} \omega_{0}\right)$, and $\tilde{R}\left(f_{\ell} \omega_{0}\right)$ we use the outputs of Christine 1-D's tape helix model and space charge reduction factor calculation. These parameter values ensure that MUSE, LATTE, and S-MUSE use the same dispersion parameters as Christine 1-D.

The set of frequencies includes two drive frequencies, the second-order products, and the third-order intermodulation (3IM) frequencies $2 f_{1}-f_{2}$ and $2 f_{2}-f_{1}$. Table II lists the frequencies and dispersion parameters (drive frequency data in bold).

The calculations are done using a fixed step fourth-order Runge-Kutta integrator.

\section{A. Power Versus Axial Position}

In Fig. 1, we plot axial power of the drive, harmonic, and 3IM frequencies for the MUSE model and LATTE. The models agree extremely well for a majority of the TWT length, but there is disagreement between the models at saturation. For a quantitative comparison of all of the models, we plot decibel difference of the models from LATTE. Figs. 2-4 show the decibel difference from LATTE for the drive frequencies, harmonics, sum frequency, and the 3IMs respectively. For each model, the frequency pair having the largest maximum deviation is represented in the figure. 
TABLE II

SIMULATION FREQUENCIES AND DISPERSION PARAMETERS

\begin{tabular}{cccc}
$f(\mathrm{GHz})$ & $\tilde{K}(\Omega)$ & $\tilde{v}_{\text {ph }}\left(\times 10^{7} \mathrm{~m} / \mathrm{s}\right)$ & $\tilde{R}$ \\
\hline 0.001 & 365.40 & 3.2845 & 0.00100 \\
1.599 & 225.13 & 2.9983 & 0.04529 \\
$\mathbf{1 . 6 0 0}$ & $\mathbf{2 2 4 . 9 8}$ & $\mathbf{2 . 9 9 8 0}$ & $\mathbf{0 . 0 4 5 3 5}$ \\
$\mathbf{1 . 6 0 1}$ & $\mathbf{2 2 4 . 8 2}$ & $\mathbf{2 . 9 9 7 7}$ & $\mathbf{0 . 0 4 5 4 0}$ \\
1.602 & 224.67 & 2.9974 & 0.04545 \\
3.200 & 42.68 & 2.6460 & 0.14945 \\
3.201 & 42.62 & 2.6459 & 0.14952 \\
3.202 & 42.57 & 2.6458 & 0.14959
\end{tabular}

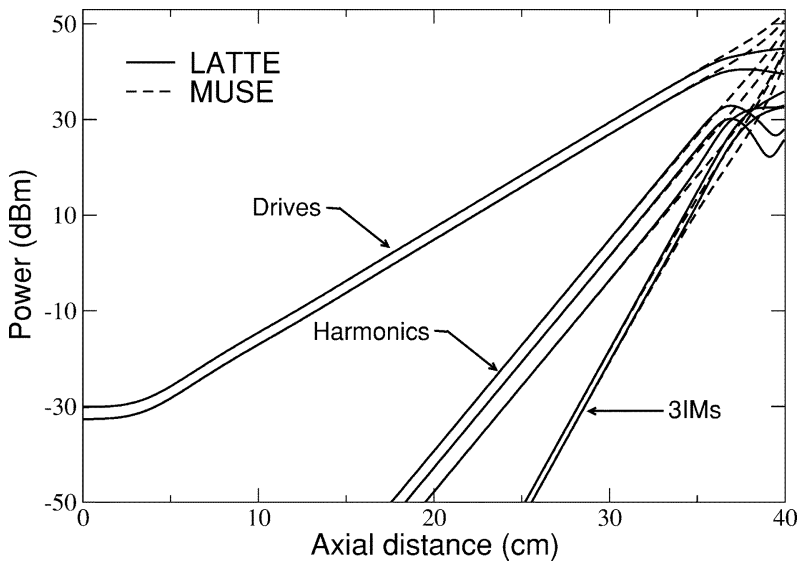

Fig. 1. Power versus axial distance for LATTE and MUSE.

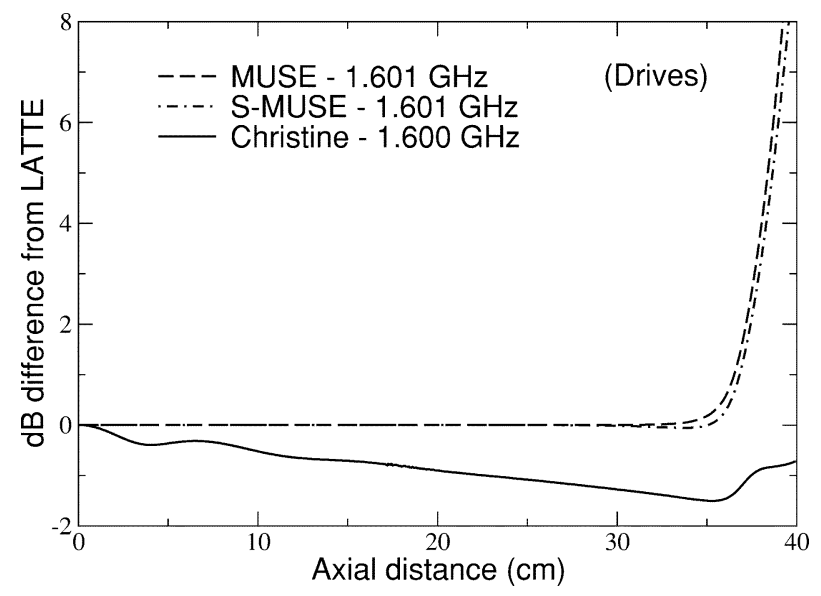

Fig. 2. Decibel difference of drive frequencies from LATTE versus axial distance.

For small $z$ values, Figs. 3 and 4 exhibit large fluctuations in the decibel difference from LATTE for the harmonics and 3IMs, respectively. However, the numbers being compared are very small and are below the numerical noise floor of the computations. The differences between the harmonics converge before the differences between the 3IMs since the harmonics need to rise above the numerical noise floor before the 3IMs may rise above the numerical noise floor. For $z=5 \mathrm{~cm}$, steady-state differences are achieved in both figures.

Inspection of Figs. 2-4 confirms that the agreement between LATTE and MUSE is very good prior to the onset of saturation (roughly $z \leq 35 \mathrm{~cm}$ ). Up to $z=30 \mathrm{~cm}$, the discrepancy is less than $0.1 \mathrm{~dB}$. The constant level of the nonlinear products

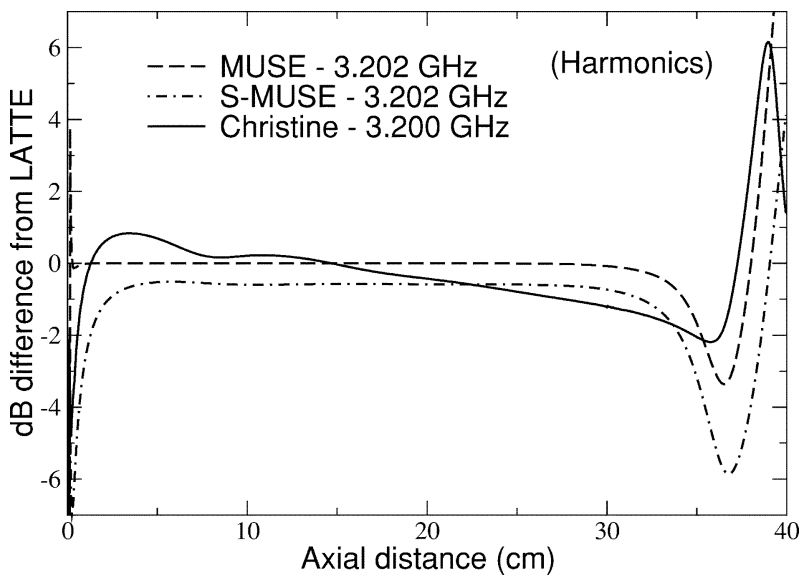

Fig. 3. Decibel difference of harmonics and sum frequency from LATTE versus axial distance.

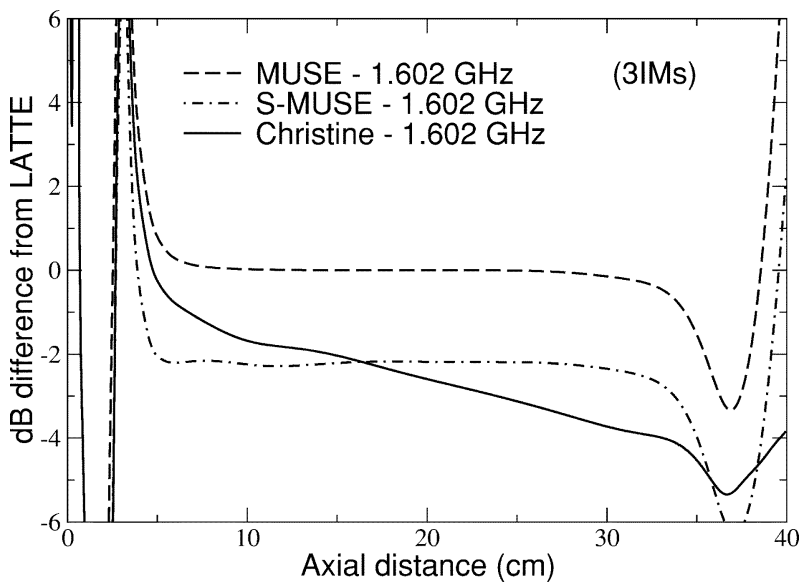

Fig. 4. Decibel different 3IMs from LATTE versus axial distance.

confirms that growth rates of LATTE, MUSE, and S-MUSE are the same, even though we see that S-MUSE is less accurate than MUSE, especially for the higher order nonlinear products.

Finally, we see the difference between LATTE and Christine $1-\mathrm{D}$ is $<2 \mathrm{~dB}, \leq 3 \mathrm{~dB}$, and $\leq 5 \mathrm{~dB}$ in the drives, harmonics, and $3 \mathrm{IMs}$, respectively for $z \leq 35 \mathrm{~cm}$. One sees from Fig. 2 that the linear growth rates of the drive frequencies are slightly different for the two models, which results in a disagreement of predicted power between the models which grows with axial distance. The difference in gain predicted by the two models is about $1.5 \mathrm{~dB}$ out of $72 \mathrm{~dB}$. The differences in the nonlinear products are likely due to the nonlinear amplification of the differences in the drive frequencies.

Since Christine 1-D has been validated experimentally [7], the disagreement between LATTE and Christine 1-D raises the question of experimental validation of LATTE and MUSE. To address this question, we studied the sensitivity of the output power on certain input parameters. For the present case, we found that less than a $1 \%$ change in beam voltage can produce a 1-dB difference in output power, and a $10 \%$ change in "smeared" relative dielectric constant can produce more than a 2-dB difference in output power. In [7], beam voltage and relative dielectric constant were changed by more than $8 \%$ from their experimental values. Therefore, we observe that the input 
parameters to our models could be changed within the same bounds used in [7] to match Christine 1-D results.

\section{B. Constant of the Motion}

Appendix I shows that the total energy density (53) is a constant of the motion for MUSE and LATTE when circuit parameters are independent of axial distance. This is confirmed in Fig. 5, which also shows the energy density for S-MUSE. Since S-MUSE is an approximation to MUSE, one would not expect (53) to be a constant of the motion. However, as seen in Fig. 5, S-MUSE predicts the value to be constant until $z=30 \mathrm{~cm}$.

Having a constant of the motion can be a useful aid in an investigation of the physics in a model. For example, while LATTE and MUSE predict the constant of the motion, MUSE does not exhibit a saturation (see Fig. 1). This suggests that the partitioning of the energy density is not consistent between the models. In Fig. 6, we see that the energy density in the circuit quantities of MUSE continues to rise where that of LATTE saturates. We also see that the beam kinetic energy density continues to decrease in MUSE where in LATTE it reaches a minimum and starts to increase. Additionally, there is a difference in the energy density in the space charge field not shown in Fig. 6. Thus, the energy density terms from the constant of the motion reveal another view of the deviation of the models. Furthermore, the constant of the motion provides a means of checking the error in numerical calculations.

\section{Electron Overtaking}

Nordsieck's Lagrangian formulation was motivated by the fact that "electrons overtake one another at or even considerably before the point along the tube where the limiting power level is obtained," [2] in which case Eulerian functions become multivalued. However, Paschke wanted to "dispel the widespread belief that, because of overtaking, the hydrodynamic model must break down at large levels" [22] with nonlinear Eulerian treatments of electron beams. ${ }^{2}$ Since LATTE and MUSE come from the same "hydrodynamic" equations, they are ideally suited to consider overtaking and its role in the deviation of MUSE from LATTE.

The Jacobian of the transformation from Lagrangian to Eulerian coordinates is (39)

$$
J=\frac{\partial \Psi}{\partial \psi} .
$$

If $J>0$ for all $\psi_{0}$ at some $z$, then the electron beam, while perhaps bunched, retains the same "disk ordering" (in time) it had at $z=0$. On the other hand, if for some $\left(z, \psi_{0}\right)$ we have $J<0$, then some disks in the beam have exchanged positions. The $z$ at which electron overtaking occurs is such that $J=0$ for exactly one value of $\psi_{0}$. Beyond this $z$ value $J\left(z, \psi_{0}\right)$ has exactly two zeroes in $\psi_{0}$ (for simple overtaking). While overtaking can be observed on a disk trajectory plot such as Fig. 7, it may not be possible to establish the exact

\footnotetext{
${ }^{2}$ The view taken in this paper is that the Eulerian and Lagrangian models are both "hydrodynamic," i.e., they describe the electron beam as a fluid. However, the functions in Lagrangian coordinates allow for the fluid to "fold" over on itself. Only when one seeks approximate numerical solutions to the Lagrangian equations does one get a "disk" model.
}

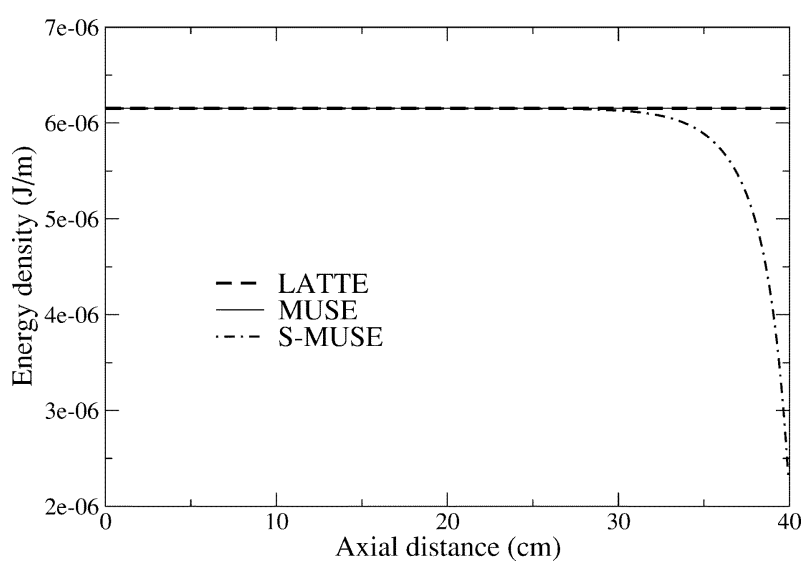

Fig. 5. Constant of the motion versus axial distance.

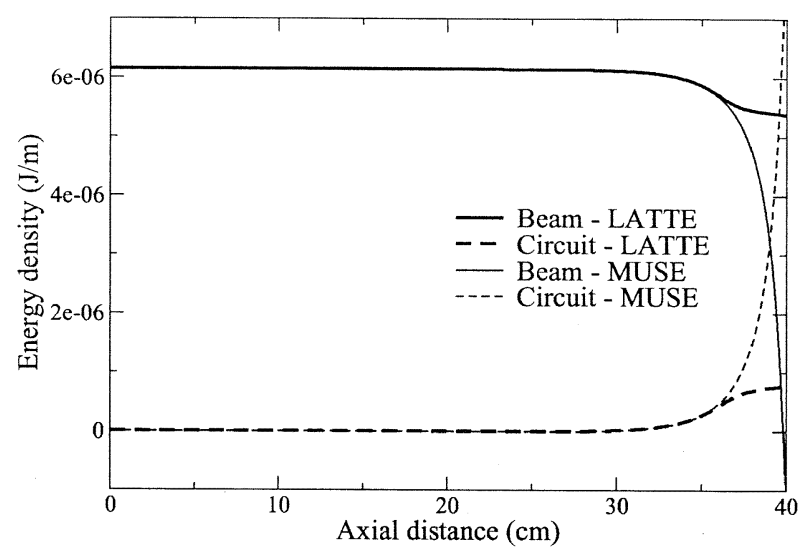

Fig. 6. Energy densities in circuit and beam versus axial distance for LATTE and MUSE. Energy density in space charge field not shown.

axial position at which overtaking first occurs. Computing $J$ such as in Fig. 8, one can precisely determine the exact axial position where the overtaking starts.

To study if there is a correlation between electron overtaking and the deviation of MUSE from LATTE, we consider a simulation of one drive tone at $f=1.6 \mathrm{GHz}$ with the drive level such that saturation occurs at $z=39.5 \mathrm{~cm}$. Detailed inspection of Fig. 8 reveals that $J=0$ for one value of $\psi_{0}$ at $z=36.43 \mathrm{~cm}$. Detailed study of Fig. 9, which shows a closeup of the point at which MUSE and LATTE deviate, reveals the position of the deviation to be roughly $z=36.3 \mathrm{~cm}$. Therefore, it seems that the deviation of MUSE and LATTE is correlated to electron overtaking.

It is also interesting that the Eulerian model of Datta et al. [23] exhibits saturation for the cases they consider. The discrepancies between the Datta model and MUSE will be a subject of future investigation. Future studies will also attempt to use recent developments in numerical analysis for computing multivalued solutions of Eulerian functions [24] to enable MUSE to simulate charge overtaking.

\section{Choosing Simulation Frequencies}

Our implementations of MUSE, S-MUSE, and LATTE allow the user to choose any frequency that is an integer multiple of the base frequency. Alternatively, there are two methods of 


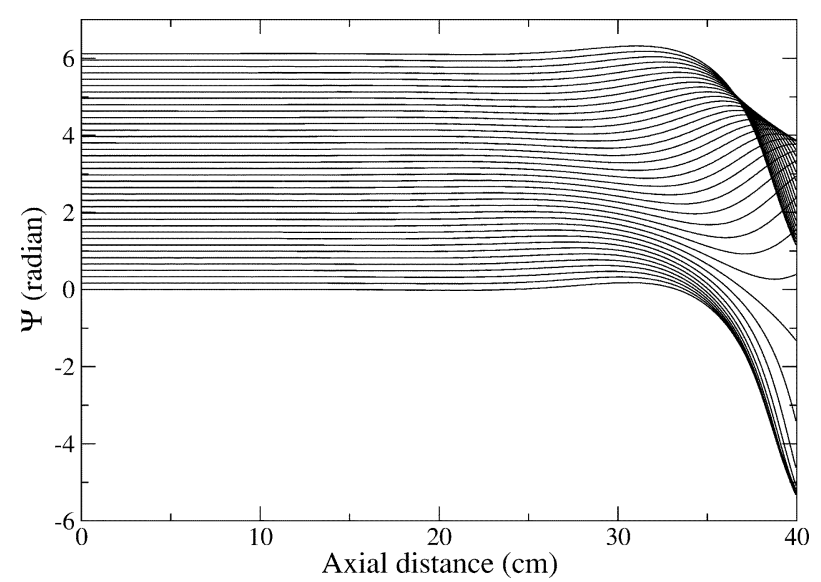

Fig. 7. Disk orbits versus axial distance.

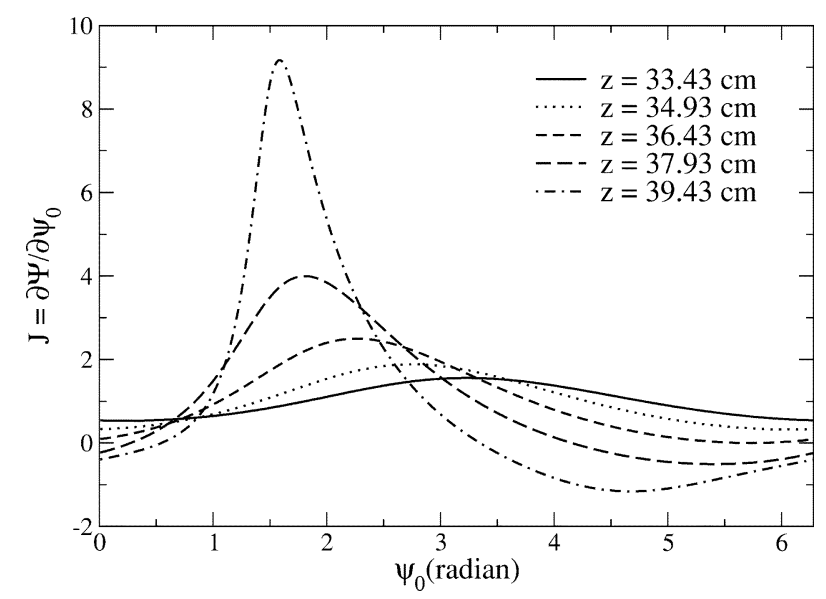

Fig. 8. Jacobian versus $\psi_{0}$ for several axial positions.

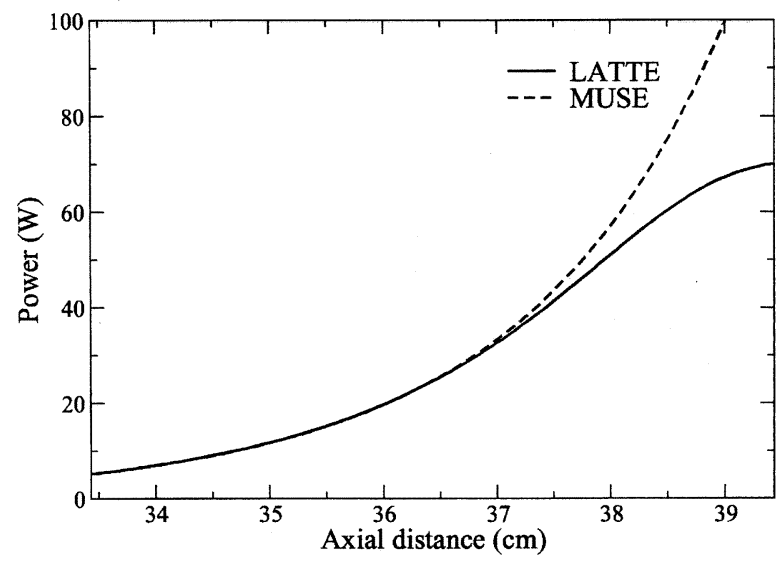

Fig. 9. Power versus axial distance for LATTE and MUSE.

choosing simulation frequencies in the Christine 1-D code. First, one can have the code create an array of frequencies between specified minimum and maximum frequencies spaced by the base frequency. This method alone, however, would make it impractical to include harmonics of closely spaced drive tones since all frequencies between the drive and the harmonic frequencies that are spaced by the difference frequency would be included. Therefore, the Christine 1-D code provides an integer $n$ which allows the user to specify an array for frequencies close to the drive frequencies only, and creates $n$ "harmonic windows" which are frequency arrays containing harmonics of the specified frequency array. The frequencies generated by the use of the harmonic window function may include additional frequencies that are not exactly harmonics of the specified array.

The implications of Christine 1-D's frequency selection method meant that in our Christine 1-D simulations, we did not include the difference frequency $f=1.0 \mathrm{MHz}$. Also, due to the use of the harmonic window function, we did include a tone at $f=3.203 \mathrm{GHz}$. First, we discuss the effect of the difference frequency, then the tone at $f=3.203 \mathrm{GHz}$ which is not a nonlinear product of the drive frequencies.

Because of the frequency convolutions in the MUSE model, to predict the evolution of a particular frequency, in principal all frequency pairs adding to the particular frequency need to be accounted for. However, in practice only the dominant contributors are considered. For this example, we included the difference frequency $f=1.0 \mathrm{MHz}$ since it had a $0.5-\mathrm{dB}$ effect on the level of the 3IM in the MUSE simulation.

To estimate the effect of leaving the difference frequency out of the Christine 1-D simulation, we ran LATTE simulations including and excluding $f=1.0 \mathrm{MHz}$. For these simulations, we observed that the level of the 3IM depends only slightly on the inclusion of the difference frequency $(<0.05 \mathrm{~dB})$. Hence, we conclude similar behavior is likely in Christine 1-D. We do not show results for the $1.0-\mathrm{MHz}$ signal since it is so far out of the bandwidth of the TWT.

For $f=3.203 \mathrm{GHz}$, we ran LATTE simulations including and excluding this frequency and found that the effect of including $f=3.203 \mathrm{GHz}$ is negligible $(<0.02 \mathrm{~dB}$ difference in 3IMs, less in other tones). Furthermore, preliminary analytic results from S-MUSE indicate that amplitudes of intermodulation frequencies are determined primarily by the amplitudes of frequencies mixing to make the product. Therefore, since $f=3.203 \mathrm{GHz}$ is not related to the drives or intermodulation frequencies, we expect that it will not have a significant effect. For the difference frequency, we saw that even though it is a nonlinear product related to the 3IM, it had a small effect on the 3IM in LATTE. An unrelated frequency can be expected to have an even smaller effect.

In sum, we conclude that even though $f=1.0 \mathrm{MHz}$ was excluded and $f=3.203 \mathrm{GHz}$ was included in the Christine 1-D simulations, it is still appropriate to compare these results to those of MUSE and LATTE.

Finally, we discuss getting dispersion data for $f=1.0 \mathrm{MHz}$ since Christine 1-D did not simulate $f=1.0 \mathrm{MHz}$ and, hence, did not generate dispersion data for it. The circuit dispersion parameters at $f=1.0 \mathrm{MHz}$ were calculated with an independent tape helix solver and the space charge reduction factor is an estimate based on the values for other frequencies. However, based on MUSE simulations, the prediction of the difference frequency does not seem to depend on the dispersion parameters at $f=1.0 \mathrm{MHz}$. This is consistent with preliminary analytic results from S-MUSE which suggest that when a nonlinear 
product frequency lies out of the linear gain-bandwidth its amplitude and growth rate are primarily determined by the drive frequencies which mix to produce it.

\section{DISCUSSION}

\section{A. Nonlinearities}

The nonlinearities of the MUSE model are different than the nonlinearities of LATTE. The MUSE model has quadratic nonlinearities that arise from the quadratic nonlinearities of the Eulerian electron beam equations. In contrast, LATTE has quadratic nonlinearities, $1 / v$ nonlinearities, and complex exponential nonlinearities. As the MUSE predictions agree with LATTE for a majority of the tube length, one can argue that the nonlinear behavior in this region can be described by quadratic nonlinearities. As discussed in Section V, the quadratic nonlinearity is easier to study analytically than the nonlinearities in LATTE.

A quadratic nonlinearity in the time domain becomes a convolution in the frequency domain, as seen in the MUSE model (18)-(22). This allows one to observe the origin of harmonic and intermodulation frequencies based on the nonlinear combinations of beam velocity and density. As a brief demonstration of the physical insight to be gained from this fact, we consider alternately linearizing $v$ and $\rho$ in the derivation of the MUSE model from (1)-(5). We use the equations resulting from the "partial linearizations" to examine whether nonlinearities involving $v$ or nonlinearities involving $\rho$ in (4) and (5) play a larger role in producing distortions.

In both partial linearizations, since (1)-(3) are linear, (18)-(20) are reproduced. If we linearize $v$ in (4) and (5), make the coordinate transformation (9), and compute Fourier coefficients, we get instead of (21) and (22)

$$
\begin{aligned}
& \frac{d \tilde{v}_{\ell}}{d z}=\frac{i f_{\ell} \omega_{0} e \tilde{K}\left(f_{\ell} \omega_{0}\right)}{m_{e} \tilde{v}_{\mathrm{ph}}\left(f_{\ell} \omega_{0}\right) u_{0}} \tilde{I}_{\ell}+\frac{e}{m_{e} u_{0}} \tilde{R}\left(f_{\ell} \omega_{0}\right) \tilde{E}_{\ell} \\
& \frac{d \tilde{\rho}_{\ell}}{d z}=-\frac{1}{u_{0}} \sum_{\substack{m, n \\
f_{m}+f_{n}=f_{\ell}}} \tilde{\rho}_{m}\left[\frac{d \tilde{v}_{n}}{d z}+\frac{i f_{n} \omega_{0}}{u_{0}} \tilde{v}_{n}\right] .
\end{aligned}
$$

Notice that $d \tilde{\rho}_{0} / d z \neq 0$.

Similarly linearizing $\rho$ reproduces (21) and from (5), one gets

$$
\begin{aligned}
\sum_{\substack{m, n \\
f_{m}+f_{n}=f_{\ell}}} \tilde{v}_{m} \frac{d \tilde{\rho}_{n}}{d z}=i f_{\ell} \omega_{0} \tilde{\rho}_{\ell} & -\frac{1}{u_{0}} \sum_{\substack{m, n \\
f_{m}+f_{n}=f_{\ell}}} i f_{n} \omega_{0} \tilde{v}_{m} \tilde{\rho}_{n} \\
& -\rho_{0}\left[\frac{d \tilde{v}_{\ell}}{d z}+\frac{i f_{\ell} \omega_{0}}{u_{0}} \tilde{v}_{\ell}\right] .
\end{aligned}
$$

In (33), it is understood that $d \tilde{\rho}_{0} / d z=0$.

Fig. 10 shows the result of integrating the MUSE model (18)-(22), the $v$-linearized equations (18)-(20), (31), (32), and the $\rho$-linearized equations (18)-(21), (33) in the $8537 \mathrm{H}$ constant pitch section for a drive frequency of $f=1.6 \mathrm{GHz}$. Linearizing the velocity reduces the level of the circuit harmonic more than linearizing the density. Therefore, the velocity has a larger role in producing the harmonic. This observation appears to be valid for many cases.

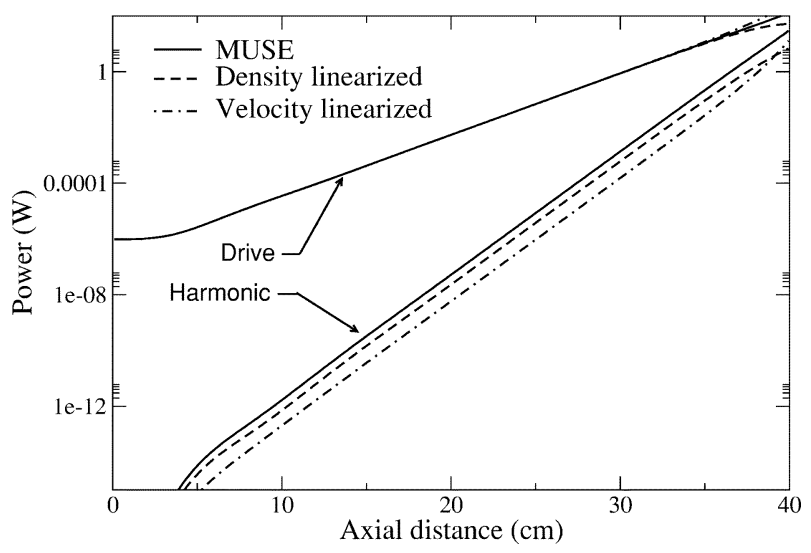

Fig. 10. Power versus axial distance for partially linearized equations.

\section{B. Dimension Scaling of LATTE and MUSE}

The computation time of systems of ordinary differential equations (ODEs) such as LATTE, MUSE, and Christine 1-D depends on the number of state variables in the simulation, i.e., the dimension of the model. (The dimension of the model is equal to the number of coupled ODEs in the system, and hence equal to the number of derivatives that must be calculated at each step of the integration.) The dimension of the MUSE model depends only on the number of frequencies; however, the set of frequencies in a simulation must be chosen with care to assure that all nonlinear product frequencies are accounted for correctly. In a disk model the majority of the dimensions are those accounting for the disks. The number of disks depends on several factors which we will discuss. To simplify the discussion, we consider the dimensions of MUSE and LATTE, noting that other disk models will have similar behavior to LATTE.

LATTE has six complex dimensions per simulation frequency plus $2 N$ real dimensions for the disks. MUSE has ten complex dimensions per simulation frequency plus five dimensions for the DC quantities.

To compare dimensions of LATTE to MUSE, one needs a formula for the number of disks based on simulation parameters. An estimate for computing the number of disks is given in the Christine 1-D documentation [5]. Depending on simulation parameters, the number of disks may need to be increased to obtain convergence of results. We provide a version of this formula applicable to LATTE here. If $N_{\text {base }}$ is a "base" number of disks, $N_{f}$ the number of tones in the simulation, and $N_{H}$ is the number of harmonics present, then a starting point for the number of disks $N$ in a simulation is given by

$$
N=1.5 N_{\text {base }} N_{f} N_{H}
$$

It is suggested that $N_{\text {base }}$ initially be chosen near 19 and that the choice of $N_{\text {base }}$ always be a prime number.

To understand the dimensional dependence on frequency of MUSE, one needs to account for the fact that to simulate a nonlinear product frequency, one may be required to simulate frequencies one is not directly interested in. For example, due to the frequency convolutions in the MUSE model, to simulate 3IMs one needs to track all pairs that sum to these frequencies. Thus, 
to simulate the 3IMs $2 f_{1}-f_{2}$ and $2 f_{2}-f_{1}$, one needs to account for $2 f_{1}, 2 f_{2}, f_{1}-f_{2}$, and $f_{2}-f_{1}$ in addition to the drive frequencies.

For the example presented, we have

$$
\begin{aligned}
N_{\text {base }} & =19 \\
N_{f} & =8 \\
N_{H} & =2 \\
N & =456 .
\end{aligned}
$$

Thus, the dimension of LATTE is 960 (912 real, 48 complex). For MUSE we have 85 complex dimensions.

As an example of using MUSE in cases with many frequencies, we include results of a simulation modeled after the noise power ratio simulations in [8], but using the lossless, constant pitch TWT parameters from Section III. The input spectrum consists of 101 evenly spaced frequencies $(0.4 \mathrm{MHz}$ spacing) with a $3 \mathrm{MHz}$ notch at the center. The total number of frequencies when accounting for all sum and difference frequencies is 402. The nonzero input amplitudes are assigned randomly between $-20 \mathrm{dBm}$ and $-30 \mathrm{dBm}$. The input and output spectra are shown in Fig. 11. Not shown in Fig. 11 is the spectrum at the sum and difference frequencies. Since the TWT simulation parameters did not include circuit loss or a sever, the output was taken at $z=26 \mathrm{~cm}$. The simulation ran for $5 \mathrm{~h}$ and $40 \mathrm{~min}$ on a 1.3-GHz Gnu/Linux PC. As yet, there has been no attempt to optimize the speed of the code.

\section{Relation to Method of Collective Variables}

In this section, we compare MUSE to a collective variable model, and we outline a method for developing a MUSE type model for free-electron lasers (FELs). Since the TWT may be described by the same "nonlinear pendulum" equations as the FEL, e.g., [26], we propose that the prescription would result in a useful multifrequency analysis and simulation tool for FELs.

The majority of the initial collective variable work was done for single frequency excitations, where "in the presence of multiple frequencies, it is extremely difficult to obtain a description of FEL dynamics in terms of a reduced set of equations involving collective variables" [27]. With some simplifications and normalizations, the MUSE model can be transformed into such a multifrequency collective variable model, applicable at least in the exponential growth region and potentially into the oscillation region.

The method of collective variables [9] involves first defining the "bunching parameter," the first collective variable, as the complex exponential average over particle phases, then differentiating the definition which results in an equation containing a higher "moment." One defines this higher moment as the next collective variable and differentiates this definition to get an equation which contains the next higher moment. At this point, a relation is employed to "close" the system of equations.

In contrast, the MUSE model recognizes the first collective variable as the Fourier coefficient of the electron beam charge density, and defines a "second collective variable" as the Fourier coefficient of the electron beam velocity. To get a multifrequency "collective variable model" using the MUSE

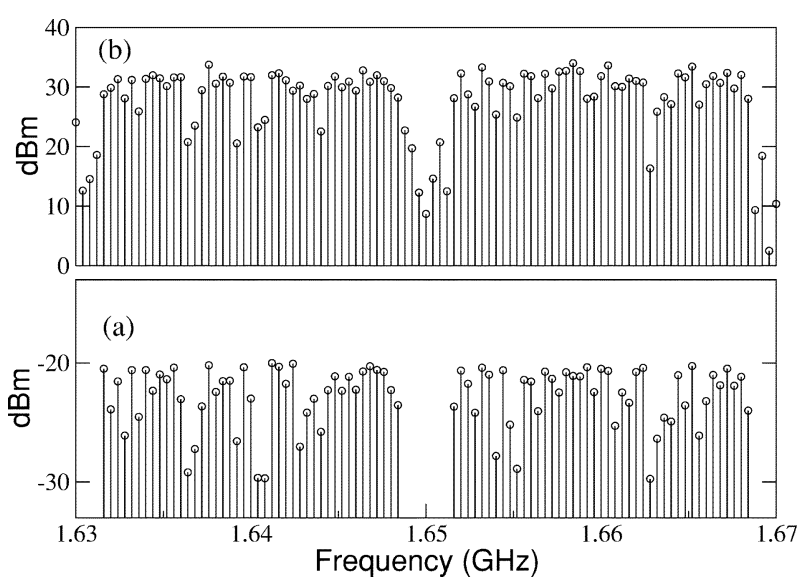

Fig. 11. (a) Input and (b) output spectra of a simulation with 402 frequencies.

theory one does a derivation similar to Section II. In particular, one uses the Eulerian electron beam equations, neglecting space charge, and a wave equation for only a forward wave. The result is a system that closely resembles (after normalization) a multifrequency collective variable model that does not need to be "closed."

Using the change of variable equations from Eulerian to Lagrangian coordinates one finds that the second collective variable of [9] is related to beam current. Thus, by taking moments the collective variable method misses having a collective variable for electron beam velocity. This is the primary difference between the approach of [9] and the MUSE approach.

\section{ANALYSIS Using S-MUSE}

The S-MUSE model has a form amenable to analysis and insights. In particular, the quadratic nonlinearity of the S-MUSE model is easier to analyze than the nonlinearities of LATTE and enables a new view of the generation of harmonics and intermodulation products.

Under suitable conditions, the S-MUSE model in the vector form (30) can be solved with a series solution

$$
\mathbf{x}=\sum_{\alpha=1}^{\infty} \mathbf{x}^{(\alpha)}
$$

where each vector $\mathbf{x}^{(\alpha)}$ satisfies a differential equation that is a linear system forced by quadratic terms formed by combinations of $\mathbf{x}^{(\beta)}$ with $\beta<\alpha$. For example, the drive frequencies are in $\mathbf{x}^{(1)}$ and they combine quadratically to force the differential equation for $\mathbf{x}^{(2)}$ so as to cause second harmonics in $\mathbf{x}^{(2)}$. These second harmonics, in turn, combine nonlinearly with the drive frequencies to force the differential equation for $\mathbf{x}^{(3)}$ so as to cause third order intermodulation frequencies in $\mathbf{x}^{(3)}$. Further intermodulation frequencies appear as the process continues. The key point is that intermodulation frequencies of interest are generated in a systematic analytic procedure by the effects of successive quadratic nonlinearities in the differential equations for $\mathbf{x}^{(\alpha)}$. By identifying the growth rates present in the equations at each stage, the maximum growth rates of harmonic and intermodulation frequencies can be predicted from formulas [28]. The results from these formulas can differ from 
the conventional rules of thumb for narrow band TWTs that predict that third-order intermodulation frequencies are three times the drive frequency.

This view from S-MUSE of the origin of harmonic and intermodulation frequencies also suggested how appropriate harmonic injections could advantageously modify intermodulation frequencies. In particular, it predicted that large reductions in intermodulation products during multifrequency drive could be realized by injection of the second harmonic at the right phase and amplitude. This effect has been confirmed by experiment [29]-[31].

\section{CONCLUSION}

We have derived a new nonlinear multifrequency Eulerian TWT model, the MUSE model. The MUSE model differs from other available nonlinear multifrequency Eulerian models in its suitability for analyzing larger numbers of input tones. The MUSE model also has some similarities with the method of collective variables in FEL theory [9], but one of the "collective variables" of the MUSE model is a Fourier coefficient of beam velocity and the MUSE model does not require a closure relation.

To enable a direct comparison with Lagrangian methods, a Lagrangian "disk" model LATTE is also derived. MUSE and LATTE show excellent agreement before saturation when simulated on a constant pitch section of the Hughes (now Boeing) $8537 \mathrm{H}$ TWT. The discrepancy between MUSE and LATTE begins at the start of electron overtaking, which can be quantified by singularity in the coordinate transformation from Lagrangian to Eulerian coordinates. Also, results for LATTE (and, hence, MUSE before saturation) for the TWT parameters used are comparable with the Christine 1-D Lagrangian simulation (Christine has been previously validated against experiment on the $8537 \mathrm{H}$ TWT [7]).

The relative contributions of different nonlinearities in the MUSE model to harmonic and intermodulation distortions are demonstrated by simulating with selected nonlinearities removed. In the constant pitch TWT example, the nonlinearities due to the velocity contribute more strongly to the formation of harmonics than the nonlinearities due to the density. In the example studied, MUSE has about one-fifth the number of equations compared with a Lagrangian model such as LATTE, but this difference in the number of equations is not sufficient to suggest a speed advantage of more than one order of magnitude in simulation of this example with MUSE. However, as the number of frequencies increases, there is potential for such a speed advantage.

The MUSE model has only quadratic nonlinearities, whereas Lagrangian models such as LATTE have more complicated nonlinearities. Indeed, a simplification of the MUSE model called S-MUSE is well suited for analytic purposes. The growth rates of S-MUSE agree with MUSE results in the example given, but there is a significant difference in the power prediction, particularly in the 3IMs $(\sim 40 \%)$. S-MUSE has the form of vector differential equations with linear and quadratic terms and we have briefly indicated how insights into harmonic and intermodulation generation, growth rates, and suppression can be pursued.

\section{APPENDIX I \\ LATTE MODEL}

This appendix details the coordinate change that derives the Lagrangian "disk" model, LATTE, from the Eulerian equations (10)-(14).

\section{A. Derivation}

The Eulerian independent variables are $(z, \psi)$, where $z$ is axial position and $\psi$ is phase. The Lagrangian independent variables are $\left(z, \psi_{0}\right)$, where $z$ is axial position and $\psi_{0}$ is the phase position of a fluid element with respect to the stream wave ${ }^{3}$ when the fluid element is at $z=0 . \psi_{0}$ and $\psi$ take values from 0 to $2 \pi$.

The transformation from Lagrangian to Eulerian coordinates is given by functions $Z$ and $\Psi$

$$
\left[\begin{array}{l}
z \\
\psi
\end{array}\right]=\left[\begin{array}{l}
Z\left(z, \psi_{0}\right) \\
\Psi\left(z, \psi_{0}\right)
\end{array}\right]
$$

$Z\left(z, \psi_{0}\right)$ is the axial position of fluid element $\psi_{0}$ at $z$ so

$$
Z\left(z, \psi_{0}\right)=z
$$

$\Psi\left(z, \psi_{0}\right)$ is the phase position of fluid element $\psi_{0}$ with respect to the stream wave at $z$.

A function $g^{\mathrm{E}}$ of Eulerian variables $(z, \psi)$ is transformed to a function $g^{\mathrm{L}}$ of Lagrangian variables $\left(z, \psi_{0}\right)$ using

$$
\begin{aligned}
g^{\mathrm{L}}\left(z, \psi_{0}\right) & =g^{\mathrm{E}}\left(Z\left(z, \psi_{0}\right), \Psi\left(z, \psi_{0}\right)\right) \\
& =g^{\mathrm{E}}\left(z, \Psi\left(z, \psi_{0}\right)\right) .
\end{aligned}
$$

The linearization of coordinate transformation (35) is the matrix

$$
\left[\begin{array}{cc}
1 & 0 \\
\frac{\partial \Psi}{\partial z} & \frac{\partial \Psi}{\partial \psi_{0}}
\end{array}\right]
$$

and its Jacobian $J$ is the determinant of (38)

$$
J=\frac{\partial \Psi}{\partial \psi_{0}} .
$$

Partial derivatives transform via

$$
\left[\begin{array}{cc}
\frac{\partial}{\partial z} & \frac{\partial}{\partial \psi}
\end{array}\right]=\left[\begin{array}{ll}
\frac{\partial}{\partial z} & \frac{\partial}{\partial \psi_{0}}
\end{array}\right]\left[\begin{array}{cc}
1 & 0 \\
\frac{\partial \Psi}{\partial z} & \frac{\partial \Psi}{\partial \psi_{0}}
\end{array}\right]^{-1}
$$

Consistent with the method of characteristics, we take

$$
\frac{\partial \Psi}{\partial z}=\frac{\omega_{0}}{u_{0}}\left(1-\frac{u_{0}}{v^{\mathrm{L}}}\right)
$$

${ }^{3}$ The "stream wave" is a hypothetical wave of frequency $\omega_{0}$ traveling with speed $u_{0}$. 
then the convective derivative [in $(z, \psi)$ coordinates] becomes

$$
v^{\mathrm{E}} \frac{\partial v^{\mathrm{E}}}{\partial z}+\omega_{0}\left(\frac{v^{\mathrm{E}}}{u_{0}}-1\right) \frac{\partial v^{\mathrm{E}}}{\partial \psi}=v^{\mathrm{L}} \frac{\partial v^{\mathrm{L}}}{\partial z} .
$$

Applying the derivative transformations in (40) to the continuity equation (14), one gets

$$
\frac{\partial \Psi}{\partial \psi_{0}} \frac{\partial \rho^{\mathrm{L}} v^{\mathrm{L}}}{\partial z}=-\omega_{0} \frac{\rho^{\mathrm{L}}}{v^{\mathrm{L}}} \frac{\partial v^{\mathrm{L}}}{\partial \psi_{0}} .
$$

Taking $\partial / \partial \psi_{0}$ of (41), one gets

$$
\frac{\partial}{\partial z} \frac{\partial \Psi}{\partial \psi_{0}}=\frac{\omega_{0}}{\left(v^{\mathrm{L}}\right)^{2}} \frac{\partial v^{\mathrm{L}}}{\partial \psi_{0}}
$$

Substitute (44) into (43) and integrate to get

$$
\frac{\partial \Psi}{\partial \psi_{0}} \rho^{\mathrm{L}} v^{\mathrm{L}}=\kappa
$$

where $\kappa$ is a constant of integration. We set $\kappa$ by using the values of $\rho^{\mathrm{L}} v^{\mathrm{L}}$ and $\partial \Psi / \partial \psi_{0}$ on the $\psi_{0}$ axis [by definition $\Psi\left(0, \psi_{0}\right)=$ $\psi_{0}$ which implies $\partial \Psi / \partial \psi_{0}\left(0, \psi_{0}\right)=1$ ] which gives finally

$$
\left|\frac{\partial \Psi}{\partial \psi_{0}}\right| \rho^{\mathrm{L}} v^{\mathrm{L}}=\rho^{\mathrm{L}}\left(0, \psi_{0}\right) v^{\mathrm{L}}\left(0, \psi_{0}\right)=\frac{I_{0}\left(\psi_{0}\right)}{A} .
$$

As a last point regarding the coordinate transformation, we change variables in an integral. Pulling the equation for the Fourier coefficient of $\rho$ back to Lagrangian coordinates (for fixed $z$ ) one gets

$$
\begin{aligned}
\tilde{\rho}_{\ell}^{\mathrm{E}} & =\frac{1}{2 \pi} \int_{2 \pi} \rho^{\mathrm{E}} e^{-i f_{\ell} \psi} d \psi \\
& =\frac{1}{2 \pi} \int_{2 \pi} \rho^{\mathrm{L}}\left|\frac{\partial \Psi}{\partial \psi_{0}}\right| e^{-i f_{\ell} \Psi\left(z, \psi_{0}\right)} d \psi_{0} \\
& =\frac{1}{2 \pi} \int_{2 \pi} \frac{I_{0}\left(\psi_{0}\right) e^{-i f_{\ell} \Psi\left(z, \psi_{0}\right)}}{A v^{\mathrm{L}}\left(z, \psi_{0}\right)} d \psi_{0}
\end{aligned}
$$

where we have used (46) to substitute for the Jacobian.

Finally, we derive LATTE from (10)-(14). Equations (48)-(50) are (18)-(20) with (47) substituted for $\tilde{\rho}_{\ell}$. Equation (14) was used to get (47). For (13), one writes $E$ and $I$ using (15) in Lagrangian coordinates. Equation (41) is also included as model equation (52). The circuit equations, space charge equation, Newton's law, and phase relation are (leaving off the superscript L)

$$
\begin{aligned}
\frac{d \tilde{V}_{\ell}}{d z}= & -\frac{i f_{\ell} \omega_{0}}{u_{0}} \tilde{V}_{\ell}-\frac{i f_{\ell} \omega_{0} \tilde{K}\left(f_{\ell} \omega_{0}\right)}{\tilde{v}_{\mathrm{ph}}\left(f_{\ell} \omega_{0}\right)} \tilde{I}_{\ell} \\
\frac{d \tilde{I}_{\ell}}{d z}= & -\frac{i f_{\ell} \omega_{0}}{\tilde{K}\left(f_{\ell} \omega_{0}\right) \tilde{v}_{\mathrm{ph}}\left(f_{\ell} \omega_{0}\right)} \tilde{V}_{\ell}-\frac{i f_{\ell} \omega_{0}}{u_{0}} \tilde{I}_{\ell} \\
& +i f_{\ell} \omega_{0} \frac{1}{2 \pi} \int_{2 \pi} \frac{I_{0}\left(\psi_{0}\right) e^{-i f_{\ell} \Psi\left(z, \psi_{0}\right)}}{v\left(z, \psi_{0}\right)} d \psi_{0}
\end{aligned}
$$

$$
\begin{aligned}
& \frac{d \tilde{E}_{\ell}}{d z}=-\frac{i f_{\ell} \omega_{0}}{u_{0}} \tilde{E}_{\ell}+\frac{1}{\epsilon_{0} A} \frac{1}{2 \pi} \int_{2 \pi} \frac{I_{0}\left(\psi_{0}\right) e^{-i f_{\ell} \Psi\left(z, \psi_{0}\right)}}{v\left(z, \psi_{0}\right)} d \psi_{0} \\
& \frac{\partial v}{\partial z}=\frac{1}{v} \sum_{\ell=-\infty}^{\infty}\left\{\frac{i f_{\ell} \omega_{0} e \tilde{K}\left(f_{\ell} \omega_{0}\right)}{m_{e} \tilde{v}_{\mathrm{ph}}\left(f_{\ell} \omega_{0}\right)} \tilde{I}_{\ell}\right. \\
&\left.+\frac{e}{m_{e}} \tilde{R}\left(f_{\ell} \omega_{0}\right) \tilde{E}_{\ell}\right\} e^{i f_{\ell} \Psi\left(z, \psi_{0}\right)} \\
& \frac{\partial \Psi}{\partial z=} \frac{\omega_{0}}{u_{0}}\left(1-\frac{u_{0}}{v}\right)
\end{aligned}
$$

These equations are valid for an arbitrary periodic electron beam modulation. Equations (51) and (52) are ordinary differential equations parameterized by $\psi_{0}$. For calculations, one represents the beam as a finite number of "disks" and there are equations (51) and (52) for each disk. In this case, the integration over $\psi_{0}$

$$
\frac{1}{2 \pi} \int_{2 \pi} \frac{I_{0}\left(\psi_{0}\right) e^{-i f_{\ell} \Psi\left(z, \psi_{0}\right)}}{v^{\mathrm{L}}\left(z, \psi_{0}\right)} d \psi_{0}
$$

becomes the sum

$$
\frac{1}{N} \sum_{j=1}^{N} \frac{I_{0}\left(\psi_{0 j}\right) e^{-i f_{\ell} \Psi\left(z, \psi_{0 j}\right)}}{v^{\mathrm{L}}\left(z, \psi_{0 j}\right)} .
$$

\section{B. Constant of the Motion}

In Lagrangian coordinates, the time averaged linear energy density is

$$
\begin{aligned}
W & =\frac{m_{e}}{e} \frac{1}{2 \pi} \int_{2 \pi} I_{0}\left(\psi_{0}\right) \frac{v}{2} d \psi_{0}+\frac{1}{4} \sum_{\ell=-\infty}^{\infty}\left\{\frac{\tilde{K}\left(f_{\ell} \omega_{0}\right)}{\tilde{v}_{\mathrm{ph}}\left(f_{\ell} \omega_{0}\right)} \tilde{I}_{\ell} \tilde{I}_{\ell}^{*}\right. \\
& \left.+\frac{1}{\tilde{v}_{\mathrm{ph}}\left(f_{\ell} \omega_{0}\right) \tilde{K}\left(f_{\ell} \omega_{0}\right)} \tilde{V}_{\ell} \tilde{V}_{\ell}^{*}-\epsilon_{0} A \tilde{R}\left(f_{\ell} \omega_{0}\right) \tilde{E}_{\ell} \tilde{E}_{\ell}^{*}\right\}
\end{aligned}
$$

The velocity integral is the time average linear beam kinetic energy density. Expressed in Eulerian coordinates this is

$$
\frac{m_{e}}{e} \frac{1}{2 \pi} \int_{2 \pi} I_{0}\left(\psi_{0}\right) \frac{v^{\mathrm{L}}}{2} d \psi_{0}=\frac{m_{e} A}{e} \frac{1}{2 \pi} \int_{2 \pi} \frac{\rho^{\mathrm{E}}\left(v^{\mathrm{E}}\right)^{2}}{2} d \psi .
$$

In the MUSE variables, this term is

$$
\frac{m_{e} A}{e} \frac{1}{2 \pi} \int_{2 \pi} \frac{\rho^{\mathrm{E}}\left(v^{\mathrm{E}}\right)^{2}}{2} d \psi=\frac{m_{e} A}{2 e} \sum_{\substack{\ell, m, n \\ f_{\ell}+f_{m}+f_{n}=0}} \tilde{\rho}_{\ell} \tilde{v}_{m} \tilde{v}_{n} .
$$

When the circuit parameters $\tilde{K}\left(f_{\ell} \omega_{0}\right), \tilde{v}_{\mathrm{ph}}\left(f_{\ell} \omega_{0}\right)$, and $\tilde{R}\left(f_{\ell} \omega_{0}\right)$ are not functions of $z$, the energy density $W$ is a constant of the motion. The most convenient way to show this 
is to compute the derivative in Lagrangian coordinates, then change the result to Eulerian coordinates. Using $v=v^{*}$

$$
\begin{aligned}
& \frac{\partial}{\partial z} 4 W=\frac{m_{e}}{e} \frac{1}{2 \pi} \int_{2 \pi} I_{0}\left(\psi_{0}\right)\left[\frac{\partial v}{\partial z}+\left(\frac{\partial v}{\partial z}\right)^{*}\right] d \psi_{0} \\
& +\sum_{\ell=-\infty}^{\infty}\left\{\frac{\tilde{K}\left(f_{\ell} \omega_{0}\right)}{\tilde{v}_{\mathrm{ph}}\left(f_{\ell} \omega_{0}\right)}\left[\frac{d \tilde{I}_{\ell}}{d z} \tilde{I}_{\ell}^{*}+\tilde{I}_{\ell} \frac{d \tilde{I}_{\ell}^{*}}{d z}\right]\right. \\
& +\frac{1}{\tilde{v}_{\mathrm{ph}}\left(f_{\ell} \omega_{0}\right) \tilde{K}\left(f_{\ell} \omega_{0}\right)}\left[\frac{d \tilde{V}_{\ell}}{d z} \tilde{V}_{\ell}^{*}+\tilde{V}_{\ell} \frac{d \tilde{V}_{\ell}^{*}}{d z}\right] \\
& \left.-\epsilon_{0} A \tilde{R}\left(f_{\ell} \omega_{0}\right)\left[\frac{d \tilde{E}_{\ell}}{d z} \tilde{E}_{\ell}^{*}+\tilde{E}_{\ell} \frac{d \tilde{E}_{\ell}^{*}}{d z}\right]\right\} \\
& =\left[\sum _ { \ell = - \infty } ^ { \infty } \left\{\frac{i f_{\ell} \omega_{0} A \tilde{K}\left(f_{\ell} \omega_{0}\right)}{\tilde{v}_{\mathrm{ph}}\left(f_{\ell} \omega_{0}\right)} \tilde{I}_{\ell} \tilde{\rho}_{\ell}^{*}\right.\right. \\
& \left.+A \tilde{R}\left(f_{\ell} \omega_{0}\right) \tilde{E}_{\ell} \tilde{\rho}_{\ell}^{*}\right\} \\
& +\sum_{\ell=-\infty}^{\infty}\left\{-\frac{i f_{\ell} \omega_{0} A \tilde{K}\left(f_{\ell} \omega_{0}\right)}{\tilde{v}_{\mathrm{ph}}\left(f_{\ell} \omega_{0}\right)} \tilde{I}_{\ell}^{*} \tilde{\rho}_{\ell}\right. \\
& \left.\left.+A \tilde{R}\left(f_{\ell} \omega_{0}\right) \tilde{E}_{\ell}^{*} \tilde{\rho}_{\ell}\right\}\right] \\
& +\sum_{\ell=-\infty}^{\infty}\left\{\frac{i f_{\ell} \omega_{0} A \tilde{K}\left(f_{\ell} \omega_{0}\right)}{\tilde{v}_{\mathrm{ph}}\left(f_{\ell} \omega_{0}\right)}\left[\tilde{I}_{\ell}^{*} \tilde{\rho}_{\ell}-\tilde{I}_{\ell} \tilde{\rho}_{\ell}^{*}\right]\right. \\
& \text { - } \left.A \tilde{R}\left(f_{\ell} \omega_{0}\right)\left[\tilde{E}_{\ell}^{*} \tilde{\rho}_{\ell}+\tilde{E}_{\ell} \tilde{\rho}_{\ell}^{*}\right]\right\}=0 .
\end{aligned}
$$

\section{APPENDIX II}

\section{S-MUSE VECTOR COMPONENTS}

This section provides details of the vector form of S-MUSE. For elements $\mathbf{x}_{\ell_{i}}, \mathbf{A}_{\ell_{i j}}$, and $\mathbf{H}_{\ell_{i} m_{j} n_{k}}$, the subscripts $(\ell, m, n)$ run from $-M$ to $M$ excluding zero, and the subsubscripts $(i, j, k)$ run from 1 to 5 . The subscript $\ell$ indexes frequencies in the set $\left\{f_{\ell}\right\}$.

We index $\mathbf{x}$ with double subscripts such that $\mathbf{x}=$ $\left[\mathbf{x}_{-M} \cdots \mathbf{x}_{-1} \mathbf{x}_{1} \cdots \mathbf{x}_{M}\right]^{\mathrm{T}}$ and $\mathbf{x}_{\ell}=\left[\mathbf{x}_{\ell_{1}} \mathbf{x}_{\ell_{2}} \mathbf{x}_{\ell_{3}} \mathbf{x}_{\ell_{4}} \mathbf{x}_{\ell_{5}}\right]^{\mathrm{T}}=$ $\left[\tilde{V}_{\ell} \tilde{I}_{\ell} \tilde{E}_{\ell} \tilde{v}_{\ell} \tilde{\rho}_{\ell}\right]^{\mathrm{T}}$.

The matrix A contains the linear parts of (25)-(29). It is block diagonal with entry $\mathbf{A}_{\ell} \equiv \mathbf{A}_{\ell \ell}$. The entries $\mathbf{A}_{\ell_{i j}}$ of the block diagonal element $\mathbf{A}_{\ell}$ are

$$
\begin{aligned}
\mathbf{A}_{\ell_{11}} & =-\frac{i f_{\ell} \omega_{0}}{u_{0}} \\
\mathbf{A}_{\ell_{12}} & =-\frac{i f_{\ell} \omega_{0} \tilde{K}\left(f_{\ell} \omega_{0}\right)}{\tilde{v}_{\mathrm{ph}}\left(f_{\ell} \omega_{0}\right)} \\
\mathbf{A}_{\ell_{21}} & =-\frac{i f_{\ell} \omega_{0}}{\tilde{K}\left(f_{\ell} \omega_{0}\right) \tilde{v}_{\mathrm{ph}}\left(f_{\ell} \omega_{0}\right)} \\
\mathbf{A}_{\ell_{22}} & =-\frac{i f_{\ell} \omega_{0}}{u_{0}} \\
\mathbf{A}_{\ell_{25}} & =i f_{\ell} \omega_{0} A
\end{aligned}
$$

$$
\begin{aligned}
\mathbf{A}_{\ell_{33}} & =-\frac{i f_{\ell} \omega_{0}}{u_{0}} \\
\mathbf{A}_{\ell_{35}} & =\frac{1}{\epsilon_{0}} \\
\mathbf{A}_{\ell_{42}} & =\frac{i f_{\ell} \omega_{0} e \tilde{K}\left(f_{\ell} \omega_{0}\right)}{m_{e} u_{0} \tilde{v}_{\mathrm{ph}}\left(f_{\ell} \omega_{0}\right)} \\
\mathbf{A}_{\ell_{43}} & =\frac{e \tilde{R}\left(f_{\ell} \omega_{0}\right)}{m_{e} u_{0}} \\
\mathbf{A}_{\ell_{52}} & =-\frac{i f_{\ell} \omega_{0} e \rho_{0} \tilde{K}\left(f_{\ell} \omega_{0}\right)}{m_{e} u_{0}^{2} \tilde{v}_{\mathrm{ph}}\left(f_{\ell} \omega_{0}\right)} \\
\mathbf{A}_{\ell_{53}} & =-\frac{e \rho_{0} \tilde{R}\left(f_{\ell} \omega_{0}\right)}{m_{e} u_{0}^{2}} \\
\mathbf{A}_{\ell_{54}} & =-\frac{i f_{\ell} \omega_{0} \rho_{0}}{u_{0}^{2}} .
\end{aligned}
$$

Entries $\mathbf{A}_{\ell_{i j}}$ not listed are zero.

The three-tensor entries $\mathbf{H}_{\ell_{i} m_{j} n_{k}}$ for $(\ell, m, n)$ are as follows.

Case 1 If $\ell, m$, and $n$ are such that $f_{\ell}=f_{m}+f_{n}$, then

$$
\begin{aligned}
& \mathbf{H}_{\ell_{4} m_{4} n_{4}}=-\frac{i f_{n} \omega_{0}}{u_{0}^{2}} \\
& \mathbf{H}_{\ell_{5} m_{2} n_{5}}=-\frac{e}{m_{e} u_{0}^{2}} \frac{i f_{m} \omega_{0} \tilde{K}\left(f_{m} \omega_{0}\right)}{\tilde{v}_{\mathrm{ph}}\left(f_{m} \omega_{0}\right)} \\
& \mathbf{H}_{\ell_{5} m_{3} n_{5}}=-\frac{e \tilde{R}\left(f_{m} \omega_{0}\right)}{m_{e} u_{0}^{2}} \\
& \mathbf{H}_{\ell_{5} m_{4} n_{4}}=\frac{\rho_{0}}{u_{0}^{3}} i f_{n} \omega_{0} \\
& \mathbf{H}_{\ell_{5} m_{4} n_{5}}=-\frac{i f_{\ell} \omega_{0}}{u_{0}^{2}} .
\end{aligned}
$$

Entries for $(i, j, k)$ not listed are zero.

Case 2 If $\ell, m$, and $n$ are such that $f_{\ell} \neq f_{m}+f_{n}$, then

$$
\mathbf{H}_{\ell_{i} m_{j} n_{k}}=0
$$

for all $(i, j, k)$.

For the $\ell$ th component of $\dot{\mathbf{x}}$, we have

$$
\dot{\mathbf{x}}_{\ell}=\mathbf{A}_{\ell} \mathbf{x}_{\ell}+\sum_{\substack{m, n \\ f_{m}+f_{n}=f_{\ell}}} \mathbf{H}_{\ell m n}\left(\mathbf{x}_{m}, \mathbf{x}_{n}\right)
$$

where the $i$ th component of the quadratic term is

$$
\begin{aligned}
\left(\sum_{\substack{m, n \\
f_{m}+f_{n}=f_{\ell}}} \mathbf{H}_{\ell m n}\left(\mathbf{x}_{m}, \mathbf{x}_{n}\right)\right)_{i} & \sum_{\substack{m, n \\
f_{\ell}=f_{m}+f_{n}}} \sum_{j=1}^{5} \sum_{k=1}^{5} \mathbf{H}_{\ell_{i} m_{j} n_{k}} \mathbf{x}_{m_{j}} \mathbf{x}_{n_{k}}
\end{aligned}
$$




\section{ACKNOWLEDGMENT}

The authors would like to thank D. Abe and J. McDonald of the Naval Research Laboratory for compiling a version of Christine 1-D that produces the space charge reduction factor as output, and for assistance running Christine 1-D simulations.

\section{REFERENCES}

[1] J. R. Pierce, "Theory of the beam-type traveling-wave tube," Proc. IRE, vol. 35, pp. 111-123, 1947.

[2] A. Nordsieck, "Theory of the large signal behavior of traveling-wave amplifiers," Proc. IRE, vol. 41, pp. 630-637, May 1953.

[3] M. E. El-Shandwily, "Analysis of multisignal traveling-wave amplifier operation," Electron Physics Lab., Univ. Michigan, Ann Arbor, Tech. Rep. 85, June 1965.

[4] A. J. Giarola, "A theoretical description for the multiple-signal operation of a TWT," IEEE Trans. Electron Devices, vol. ED-15, pp. 381-395, June 1968.

[5] T. M. Antonsen, Jr. and B. Levush, "CHRISTINE: A multifrequency parametric simulation code for traveling wave tube amplifiers," NRL Memo Rep. NRL/FR/6840-97-9845, 1997.

[6] _ - "Traveling-wave tube devices with nonlinear dielectric elements," IEEE Trans. Plasma Sci., vol. 26, pp. 774-786, June 1998.

[7] D. K. Abe, M. T. Ngô, B. Levush, T. M. Antonsen, Jr., and D. P. Chernin, "A comparison of L-band helix TWT experiments with CHRISTINE, a 1-D multifrequency helix TWT code," IEEE Trans. Plasma Sci., vol. 28, pp. 576-587, June 2000.

[8] P. N. Safier, D. K. Abe, T. M. Antonsen, Jr., B. G. Danly, and B. Levush, "Simulation of noise-power ratio with the large-signal code CHRISTINE," IEEE Trans. Electron Devices, vol. 48, pp. 32-37, Jan. 2001.

[9] R. Bonifacio, F. Casagrande, and L. De Salvo Souza, "Collective variable description of a free-electron laser," Phys. Rev. A, Gen. Phys., vol. 33, no. 4, pp. 2836-2839, 1986.

[10] J. L. Putz, "Nonlinear phenomena in traveling-wave amplifiers," Electronics Res. Lab., Stanford Univ., Tech. Rep. 37, Oct. 1951.

[11] W. R. Curtice, "First-order nonlinear effects in travelling-wave tubes," Proc. Inst. Elect. Eng., vol. 114, no. 8, pp. 1048-1050, Aug. 1967.

[12] H. Sobol, "Modulation characteristics of O-type electron stream devices," Electron Physics Lab., Univ. of Michigan, Ann Arbor, Tech. Rep., Oct. 1957.

[13] R. W. DeGrasse, "Frequency mixing in microwave beam-type devices," Stanford University, Electron Devices Lab., Stanford Electronics Lab. Tech. Rep. 386-2, July 1958.

[14] S. K. Datta, P. K. Jain, M. D. Raj Narayan, and B. N. Basu, "Nonlinear Eulerian hydrodynamical analysis of helix traveling-wave tubes," IEEE Trans. Electron Devices, vol. 45, pp. 2055-2062, Sept. 1998.

[15] — , "Nonlinear Eulerian hydrodynamical analysis of helix traveling-wave tubes for harmonic generation and its control," IEEE Trans. Electron Devices, vol. 46, pp. 420-426, Feb. 1999.

[16] S. K. Datta, P. K. Jain, and B. N. Basu, "Control of IM3 distortion in helix TWTs by harmonic injection-An Eulerian hydrodynamical study," IEEE Trans. Electron Devices, vol. 48, pp. 62-66, Jan. 2001.

[17] J. W. Gewartowski and H. A. Watson, Principles of Electron Tubes. Princeton, NJ: Van Nostrand, 1965.

[18] R. G. E. Hutter, Beam and Wave Electronics in Microwave Tubes. Princeton, NJ: Van Nostrand, 1960.

[19] A. S. Gilmour, Jr., Principles of Traveling Wave Tubes. Norwood, MA: Artech House, 1994.

[20] W. H. Press, S. A. Teukolsky, W. T. Vetterling, and B. P. Flannery, Numerical Recipes in C: The Art of Scientific Computing. Cambridge, U.K.: Cambridge Univ. Press, 1997.

[21] V. Srivastava and S. N. Joshi, "Improved nonlinear model for multisignal analysis of helix TWTs," Proc. Inst. Elect. Eng., vol. 139, no. 2, pp. 129-134, 1992

[22] F. Paschke, "Nonlinear theory of a velocity-modulated electron beam with finite diameter," RCA Rev., vol. 21, pp. 53-74, 1960.

[23] S. K. Datta, P. K. Jain, and B. N. Basu, "Third-order saturation effects in a helix traveling-wave tube under Eulerian approximations," Microwave Opt. Technol. Lett., vol. 16, no. 6, pp. 345-349, 1997.

[24] X. Li, J. G. Wöhlbier, S. Jin, and J. H. Booske, "An Eulerian method for computing multi-valued solutions of the Euler Poisson equations and application to wave-breaking in klystrons," Phys. Rev. E., submitted for publication.

[25] T. M. Antonsen, Jr., private communication, Oct. 2001.
[26] J. G. Wöhlbier, "Modeling and analysis of a traveling wave tube under multitone excitation," M.S. thesis, Univ. Wisconsin, Madison, 2000.

[27] A. Bhattacharjee, S. Y. Cai, J. W. Dodd, and T. C. Marshall, "Optical spikes in a free electron laser: Theory and experiment," Nucl. Instr. Meth. A, vol. 304, pp. 99-103, 1991.

[28] J. G. Wöhlbier, I. Dobson, and J. H. Booske. "Generation and growth rates of nonlinear distortions in a traveling wave tube". Phys. Rev. E [Online] Available: http://ojps.aip.org/dbt/dbt.jsp

[29] O. Sauseng and W. E. Garrigus, "Reduction of intermodulation distortion with harmonic injection for wideband travelling-wave tubes," in IEDM Tech. Dig., 1975, pp. 411-414.

[30] M. A. Wirth, A. Singh, J. E. Scharer, and J. H. Booske, "Third-order intermodulation reduction by harmonic injection in a TWT amplifier," IEEE Trans. Electron Devices, vol. 49, pp. 1082-1084, June 2002.

[31] C. Armstrong, private communication, Oct. 2001.

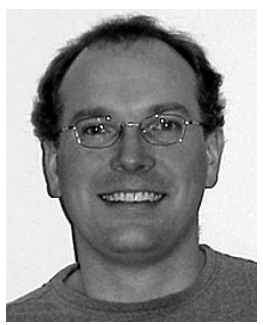

John G. Wöhlbier (S'96) is working toward the $\mathrm{Ph} . \mathrm{D}$. degree in nonlinearities in traveling wave tubes.

His research interests include microwave vacuum electronics, plasma physics, fluid dynamics, dynamical systems, and applied math.

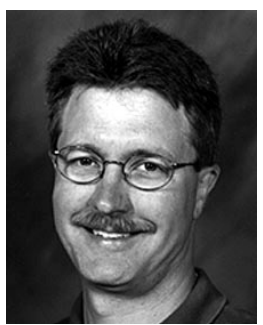

John H. Booske (S'82-M'85-SM'93) received the $\mathrm{Ph} . \mathrm{D}$. degree in nuclear engineering from the University of Michigan, Ann Arbor, in 1985.

From 1985 to 1989 , he was a Research Scientist at the University of Maryland, Baltimore, leading studies of magnetically confined hot ion plasmas and high-average power millimeter-wave free electron lasers. In 1990, he joined the faculty of the Department of Electrical and Computer Engineering, University of Wisconsin, Madison. His research activities and interests have included high-power, microwave vacuum electron devices, electron beam physics, microwave-plasma interactions, microwave-dielectric interactions, and biomedical applications of microwave radiation. His recent contributions in vacuum electronics include the development of stable periodic magnetic focusing of sheet electron beams. His current research interests include fundamental nonlinear and dispersive phenomena in helix traveling wave tubes and micromachined TWTs for millimeter wave and THz-regime applications. From 1994 to 1999, he was a Thrust Area Leader within the Center for Plasma-Aided Manufacturing, University of Wisconsin, where he investigated and developed a variety of energetic ion plasma surface modification processes for metal corrosion protection, ULSI semiconductor shallow doping, and ionized metal vapor deposition. During the past nine years, he has led a research investigation of linear and nonlinear interactions between microwave radiation and ionic crystalline ceramics. This included the experimental discovery of a nonlinear mechanism by which microwave radiation accelerates ionic diffusion in materials. His recent work includes exploring the role of this mechanism to enhance dopant activation in rapid microwave annealing of ultrashallow-implanted silicon wafers. $\mathrm{He}$ is currently Co-Principal Investigator of a Multiuniversity Research Initiative (MURI) Consortium funded by the U.S. Department of Defense to pursue basic enabling research for the next generation of innovations in microwave vacuum electronics.

Dr. Booske has won numerous awards for his teaching and investigation of innovative teaching techniques.

Ian Dobson (SM'98) received the B.A. degree in mathematics from 1978 Cambridge University, Cambridge, U.K., in 1978, and the Ph.D. degree in electrical engineering from Cornell University, Ithaca, NY, in 1989.

From 1978 to 1983, he was a Systems Analyst with EASAMS, Ltd., Surrey, U.K. In 1989, he joined the University of Wisconsin-Madison, where he is now a Professor in electrical and computer engineering. His current research interests include applications of nonlinear dynamics, electric power system instabilities, power electronics, and self-organized criticality. 